\title{
University Educator and Staff Well-being and Common Mental Health Symptoms during the COVID- 19 Pandemic in the Philippines
}

Justin Vianey M. Embalsado (Corresponding author), John lan Wilzon T. Dizon, Airajoy Keynn S. Medina, Lee Vergel S. Estacio, Beatriz C. Balilu, Mary Anne Joseph T. Montoya, Reymond Neal C. Cruz, Cherry Lou M. De Ala, Olga Angelinetta P. Tulabut, Roger S. Mangalus, Abigail B. Gonzales, Jonee O. Miranda, June R. De Leon

Angeles University Foundation

Philippines

embalsado.justinvianey@auf.edu.ph

\begin{abstract}
Educators and staff adapted to work-from-home setup amidst the covid-19 pandemic. The transition to full-online classes and services leads to poor mental health. The current study explored the association of educator and staff personal characteristics, well-being, and mental health. 326 university employees completed the demographic profile, mental health, and well-being scales. Various hierarchical regression was conducted to determine if personal characteristics and well-being predict common mental health symptoms (depression, anxiety, and stress). Series of multivariate analyses of variance (MANOVA) was conducted to determine the difference between the levels of mental health symptoms according to mental health category, and personal characteristics. The results support the hypothesis with psychological and emotional well-being inversely predicting depression, anxiety, and stress. However, social well-being failed to serve as a significant determinant of common mental health symptoms. MANOVA obtained a significant difference with common mental health symptoms and mental health category and personal characteristics.
\end{abstract}

Keywords: Employee well-being; mental health symptoms; personal characteristics.

Received 20 October 2021/Accepted 28 February 2022 @Author all rights reserved

\section{Introduction}

COVID-19 pandemic caused a drastic shift in the academic, force faculty, staff, and administrators to adopt a work-from-home setup. These changes disrupted the daily life, habits, and sleeping patterns of employees that heightened their depression, anxiety, and stress levels (Banna et al., 2020; Majumdar et 
al., 2020; Tan et al., 2020). Amidst the increasing risk of developing depression, anxiety, and stress caused by the quarantine implementation and disruption of lifestyle educational institutions continued to balance academic life and infection control (Banna et al., 2020; Cleland et al., 2020; Paulino et al., 2020; Tan et al., 2020). To continue delivering the instructions, universities are expected to create support technologies to continue teaching and learning (Van et al., 2010). However, aside from the work-related worries, educators and staff also face issues with their life, and families (Cleland et al., 2020).

Exploring the immediate effect of COVID-19 on the mental health of educators and staff can help scholars and practitioners understand their concerns and implement programs (Roy et al., 2020; Cleland et al., 2020). Existing literature on Covid and university employee and educator mental health has been limited with the understanding of depression, anxiety, and stress (i.e. Majumdar et al., 2020; Paulino et al., 2020; Talidong \& Toquero, 2020). There is a lack of literature that explore different kinds of well-being (psychological, social, and emotional), personal characteristics (marital status, employee status, employee position, gender, marital status), and mental health (depression, anxiety, and stress) of university educator and employee during the pandemic (Atiles et al., 2021; Cleland et al., 2020; Talidong \& Toquero, 2020). Thus, this study explored the influence of personal characteristics, well-being, and mental health issues of educators and staffs during the COVID- 19 pandemic.

Increasing Risk of Mental IIIness

The disruption on the daily caused by COVID-19 heightened the development of depression, anxiety, and stress within universities (Majumdar et al., 2020; Paulino et al., 2020). Developing poor mental health can hinder work productivity affecting instructional support and quality of universities (Berndt et al., 2000; Hindman \& Bustamante, 2019; Plaisier, 2007; Preece et al., 2006; Roberts et al., 20I6).

\section{Anxiety}

Educators and staff feel anxious about teaching, learning, selection, assessment, and educational needs (Cleland et al., 2020). They deal with work-related anxiety by practicing virtual learning, communicating with the professional community, adhering to quarantine requirements, and engaging with purposeful 
activities (Talidong \& Toquero, 2020; Cleland et al., 2020; Van et al., 2010). Several feel anxious during the crisis, some overextended their teaching and making them feel exhausted (Cleland et al., 2020).

\section{Depression}

The heightened levels of depression symptoms can hinder work productivity (Preece et al., 2006). Depressive symptoms of educators can influence the support and instructional quality they provide (Hindman \& Bustamante, 2019; Roberts et al., 2016). Access and guidance to curriculum plans and implementation can help educators adapt and decrease depressive symptoms (Hindman \& Bustamante, 2019). The shift to online classes created new educational needs that can increase the risk for educators and staff to develop symptoms of depression (Cleland et al., 2020; Tan et al., 2020).

\section{Stress}

The disruption in education caused by COVID-19 led to a reported increase in stress levels (Banna et al., 2020; Paulino et al., 2020). Other than the usual occupational stress of educators from workload management and student behavior (Ferguson et al., 20I2), the pandemic forced a shift to online classes creating different accommodations in education (Atiles et al., 2021; Cleland et al., 2020). For example, the transition to online classes requires the use of information and communication technology for teaching and administrative task (Kosir et al., 2020). To effectively adapt to these needs educators have to receive online teaching training, obtain access to technology, communicate with parents, and abundant access to educational materials (Atiles et al., 202I).

\section{Employee Mental Health}

Despite this evident increase in reports of common mental health symptoms (i.e., anxiety, depression, and stress) during the pandemic, exploring the role of positive mental health amidst the pandemic is equally important (Arslan et al., 2020; Yamaguchi et al., 2020). In the tripartite mental health model, Keyes (2009) argued that it takes a combination of psychological, emotional, and social well-being to be considered mentally healthy. He also proposed that individuals with combined high levels of these three dimensions are distinguished to be "flourishing" in life while those with combined low levels of these 
three are deemed to be "languishing" in life. Individuals who are neither "flourishing" nor "languishing" are identified as "moderately mentally healthy".

\section{Psychological Well-being}

The first of the three dimensions is psychological well-being (PWB), which is defined as the subjective evaluation of an individual's optimal functioning (Westerhof \& Keyes, 2010). PWB further consists of six basic elements of positive functioning which are deemed important for striving to become a better person and to realize one's potential (Ryff, 1989; Ryff \& Keyes, 1995). These six basic elements are selfacceptance, purpose in life, autonomy, positive relations with others, environmental mastery, and personal growth. Collectively, these elements of PWB are centered on an individual's reflection of his or her optimal functioning and fulfillment.

\section{Emotional Well-being}

Another essential component of the tripartite mental health model is emotional well-being (EWB) which is regarded as a balance of one's experience of positive and negative affect, and an overall cognitive judgment of satisfaction with life (Langeland, 2014). Throughout the years, researchers have been interested in the importance of assessing the impact of many different stressors on a person's EWB which could later aid the development of various mental health interventions. As mentioned by Restubog et al. (2020), the COVID-19 pandemic does not only pose significant challenges to one's work and career, and everyday activities but it also does have long-term implications on individuals' EWB. However, it must be noted that initial feelings of distress and anxiety are considered to be both normal under such circumstances. For instance, changes in mood, concentration, irritability, reduced productivity, and the like are possibly some of the repercussions brought by the stress of experiencing the pandemic (Vinkers et al., 2020).

\section{Social Well-being}

One of the components of the tripartite mental health model is social well-being (SWB). It was defined by Keyes (1998) as the perceptions and experiences of people in different social circumstances which 
also include our responses to social challenges. It also refers to an individual's satisfaction with the society to which he belongs. Furthermore, Keyes (1998) identified five dimensions of SWB, I The first dimension is social integration referring to a person's evaluation of the quality of relationships in the society. Another is social acceptance; it is a person's acceptance of other people as well as having the confidence and comfort to interact with others. The person's evaluation of worth in society is called social contribution while the belief in the possible progress of society is social actualization. Lastly, social coherence refers to the person's appraisal of society.

\section{Present Study}

The present study intends to assess the personal characteristics, common mental health symptoms, and well-being of Filipino university staff and educators during the lockdown. The objectives investigated a) if the personal characteristics and well-being determine the development of common mental health symptoms, and b) explore the possible differences between the levels of common mental health symptoms according to their well-being categories (positive mental health categories: languishing, flourishing, moderate mental health), and personal characteristics (marital status, employee status, employee position, gender, marital status).

\section{Method}

\section{Participants and Procedure}

Data were collected from August to December 2020 in the Philippines, via an online survey. The survey link was anonymously sent by email using the university mailing list to faculties, staff, and admin of one university in the Philippines. The online survey included the informed consent, demographic profile, and survey forms. The informed consent explained that participation in the study is voluntary and, they can withdraw at any point. There was also no foreseen risk. However, the survey items require recall of previous experiences regarding mental health and work experience that may bring discomfort. There are no incentives or remuneration in completing the survey. All information in the study will be stored in a secured drive using the university account for at least 5-years. 


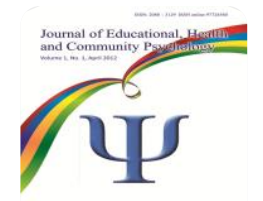

Journal of Educational, Health and Community

Psychology (Vol 11, No 1, 2022 E-ISSN 2460-8467)

In the survey, the final sample was 326 staff and educators. Most of the participants were 205 females $(62.9 \%)$, and were aged 21 to $7 \mathrm{I}(M=37, \mathrm{SD}=10.789)$. Most of the participants have expressed normal levels of depression (I72, 52.8\%), anxiety (I3I, 40.2\%), and stress (225, 69\%). While $216(63 \%)$ reported flourishing mental health (Table I).

\section{Measures}

Depression, Anxiety, and Stress Scale (DASS-2I) is a self-report scale consisting of 21 -items that ask the participants to rate how they are feeling for the past week (Lovibond \& Lovibond, 1995). DASS-2I is composed of 3 factors with 7-items for each factor, depression (I couldn't seem to experience any positive feeling at all), anxiety (I was aware of dryness of my mouth), and stress (I found it hard to wind down). It follows a 4-point Likert format from 0 (did not apply to me) to 3 (applied to me very much or most of the time). Depression, Anxiety, and Stress scales respectively obtained good interrim internal consistency of $.905,817$, and .901 .

Mental Health Continuum Short Form (MHC-SF) was used to explore employee well-being. It is a selfreport, I4-item questionnaire, using a 6-point Likert scale ranging from “Never” to "Every Day”. The 3 subscales are represented by prototypical items of different well-being constructs; psychological wellbeing (that your life has a sense of direction or meaning to it, 6-items), emotional well-being (Interested in Life, 3-items), and social well-being (that you had something important to contribute to society, 5items) (Keyes, 2009). Psychological, Emotional, and Social Well-being respectively obtained good interim internal consistency of $.912, .865$, and .879 .

\section{Results}

The descriptive statistics among the participant's demographic information was be computed followed by a correlation between variables. Series of hierarchical regression was conducted with depression, anxiety, and stress as the dependent variable. In step I, age, sex, employee position, employee status, and marital status were included. Step 2 included psychological, social, and emotional well-being. A 
multivariate analysis of variance was used to determine if there is a significant difference among the levels of depression, anxiety, and stress vary according to the well-being category (positive mental health categories: flourishing, moderate, and languishing) and personal characteristics (marital status, employee status, employee position, gender, marital status).

Table I

Demographic Profile $(\mathrm{N}=326)$

\begin{tabular}{|c|c|c|}
\hline \multicolumn{3}{|l|}{ Age } \\
\hline$M$ & $S D$ & Range \\
\hline 37 & 10.789 & $2|-7|$ \\
\hline \multicolumn{3}{|c|}{ Number of Children } \\
\hline$M$ & $S D$ & Range \\
\hline 1.206 & 1.426 & $0-6$ \\
\hline \multicolumn{3}{|c|}{ Number of People in the Household } \\
\hline M & $S D$ & Range \\
\hline 4.632 & 1.9 & $0-12$ \\
\hline \multicolumn{3}{|l|}{ Sex } \\
\hline & Frequency & Percentage \\
\hline Male & 121 & 37.1 \\
\hline Female & 205 & 62.9 \\
\hline Total & 326 & 100 \\
\hline \multicolumn{3}{|l|}{ Marital Status } \\
\hline & Frequency & Percentage \\
\hline Common-Law & 4 & 1.2 \\
\hline Legally Separated & 3 & .9 \\
\hline Married & 161 & 49.4 \\
\hline Never Married & 146 & 44.8 \\
\hline Separated & 6 & 1.8 \\
\hline Widowed & 6 & 1.8 \\
\hline Total & 326 & 100 \\
\hline \multicolumn{3}{|l|}{ Position } \\
\hline & Frequency & Percentage \\
\hline Admin & 20 & 6.1 \\
\hline Faculty & 228 & 69.9 \\
\hline NTP & 78 & 23.9 \\
\hline Total & 326 & 100 \\
\hline
\end{tabular}




\begin{tabular}{|c|c|c|}
\hline \multicolumn{3}{|l|}{ Employment Status } \\
\hline & Frequency & Percentage \\
\hline Full-time Contractual & 4 & 1.2 \\
\hline Full-time Probationary & 127 & 39 \\
\hline Full-time Regular & 189 & 58 \\
\hline Part-time & 6 & 1.8 \\
\hline Total & 326 & 100 \\
\hline Depression & Frequency & $\%$ \\
\hline Normal & 172 & 52.8 \\
\hline Mild & 54 & 16.6 \\
\hline Moderate & 52 & 16 \\
\hline Severe & 28 & 8.6 \\
\hline Extremely Severe & 20 & 6.1 \\
\hline Total & 326 & 100 \\
\hline Anxiety & Frequency & $\%$ \\
\hline Normal & 131 & 40.2 \\
\hline Mild & 28 & 8.6 \\
\hline Moderate & 82 & 25.2 \\
\hline Severe & 32 & 9.8 \\
\hline Extremely Severe & 53 & 16.3 \\
\hline Total & 326 & 100 \\
\hline Stress & Frequency & $\%$ \\
\hline Normal & 225 & 69 \\
\hline Mild & 32 & 9.8 \\
\hline Moderate & 36 & 11 \\
\hline Severe & 23 & 7.1 \\
\hline Extremely Severe & 10 & 3.1 \\
\hline Total & 326 & 100 \\
\hline \multicolumn{3}{|c|}{ Mental Health Continuum Classifications for all Participants } \\
\hline & Frequency & $\%$ \\
\hline Flourishing & 216 & 66.3 \\
\hline Moderate Mental Health & 98 & 30.1 \\
\hline Languishing & 12 & 3.7 \\
\hline Total & 326 & 100 \\
\hline
\end{tabular}

Association Between Common Mental Health Symptoms and Well-being

All correlations (see table 2) between the variables are significant. Well-being variables obtained strong and positive associations with each other. While common mental health symptoms obtained moderate 


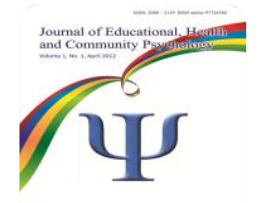

to a strong negative association with well-being variables. While all mental health symptoms have positive and strong associations with each other.

Table 2

Mental Health, Depression, Anxiety, and Stress Intercorrelations

\begin{tabular}{|c|c|c|c|c|c|c|}
\hline & I & 2 & 3 & 4 & 5 & 6 \\
\hline $\begin{array}{l}\text { I. Emotional } \\
\text { Well-being }\end{array}$ & - & & & & & \\
\hline $\begin{array}{l}\text { 2. Social } \\
\text { Well-being }\end{array}$ & $.765^{* * *}$ & - & & & & \\
\hline $\begin{array}{l}\text { 3. Psychological } \\
\text { Well-being }\end{array}$ & $.790 * * *$ & $.811 * * *$ & - & & & \\
\hline 4. Depression & $-.683 * * *$ & $-.670 * * *$ & $-.747 * * *$ & - & & \\
\hline 5.Anxiety & $-.486 * * *$ & $-.444 * * *$ & $-.507 * * *$ & $.75 I * * *$ & - & \\
\hline 6.Stress & $-.662 * * *$ & $-.605 * * *$ & $-.682 * * *$ & $.884 * * *$ & $.823 * * *$ & - \\
\hline$M$ & 11.635 & $|7.68|$ & 23.478 & 10.356 & 10.668 & II.785 \\
\hline Sd & 2.993 & 5.397 & 5.465 & 9.105 & 8.555 & 9.275 \\
\hline$\alpha$ & .865 & .879 & .912 & .905 & .817 & .901 \\
\hline
\end{tabular}

Employee Well-being as Determinants of Common Mental Health Symptoms

To test the association between common mental health symptoms and well-being various hierarchical regression analyses were conducted. Step I, personal characteristics of the employees were entered, at Step 2 well-being variables were entered. Personal characteristics were entered at step I to examine if they can influence the development of common mental health symptoms. While well-being variables were entered at step 2 to determine if the employees' current well-being affects the manifestation of common mental health symptoms (Table 3).

Only the employment status at step I in predicting depression obtained significant results with all personal characteristics. Well-being variables predicted depression, anxiety, and stress aside from social 


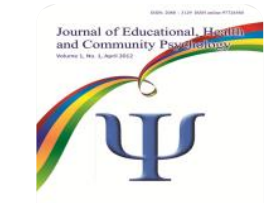

\section{Journal of Educational, Health and Community \\ Psychology (Vol 11, No 1, 2022 E-ISSN 2460-8467)

well-being. Significant accounted variance changes occurred with the inclusion of well-being variables in the analyses.

Table 3

Summary of Regression Coefficients

\begin{tabular}{|c|c|c|c|c|c|c|}
\hline \multirow[t]{2}{*}{ Variable } & \multicolumn{2}{|l|}{ Depression } & \multicolumn{2}{|l|}{ Anxiety } & \multicolumn{2}{|l|}{ Stress } \\
\hline & B & SE & $B$ & SE & B & SE \\
\hline \multicolumn{7}{|l|}{ Step I } \\
\hline Sex & -.185 & $1.07 \mid$ & -.915 & 1.023 & -.269 & 1.102 \\
\hline Marital Status & -.459 & .371 & .262 & .354 & -.347 & .381 \\
\hline $\begin{array}{l}\text { Employment } \\
\text { Status }\end{array}$ & $1.601 * *$ & .557 & $.44 I$ & .532 & 1.099 & .573 \\
\hline Position & -.087 & .975 & .168 & .931 & .406 & 1.003 \\
\hline \multicolumn{7}{|l|}{ Step 2} \\
\hline Sex & .325 & .710 & -.546 & .882 & .228 & .808 \\
\hline Marital Status & .072 & .248 & .582 & .307 & .147 & .282 \\
\hline $\begin{array}{l}\text { Employment } \\
\text { Status }\end{array}$ & .509 & .371 & -.266 & .461 & .078 & .423 \\
\hline Position & .338 & .648 & .468 & .804 & .789 & .737 \\
\hline $\begin{array}{l}\text { Emotional Well- } \\
\text { being }\end{array}$ & $-.631 * *$ & .192 & $-.598 *$ & .238 & $-.599 * *$ & .218 \\
\hline Social Well-being & -.188 & .112 & -.053 & .139 & -.148 & .127 \\
\hline $\begin{array}{l}\text { Psychological } \\
\text { Well-being }\end{array}$ & $-.809 * * *$ & .116 & $-.505^{* *}$ & .144 & $-.780 * * *$ & .132 \\
\hline Step I R2 & .026 & & .008 & & .013 & \\
\hline Step $2 R^{2}$ & .580 & & .279 & & .480 & \\
\hline$\Delta \mathrm{R}^{2}$ & .554 & & .271 & & .467 & \\
\hline
\end{tabular}

Differences Between Common Mental Health Symptoms According to Positive Mental Health Categories Employee mental health status, employment status, position, and marital status obtained significant differences according to their depression, anxiety, and stress levels (table 5). 


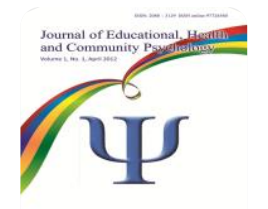

\section{Journal of Educational, Health and Community \\ Psychology (Vol 11, No 1, 2022 E-ISSN 2460-8467)}

Table 4

Split Descriptive Statistics of Common Mental Health Symptoms according to Mental Health, Employment Status, Employee Position, and Marital Status

\begin{tabular}{|c|c|c|c|c|}
\hline & Mental Health & $M$ & $S D$ & $N$ \\
\hline \multirow[t]{4}{*}{ Depression } & Languishing & 30.167 & 9.628 & 12 \\
\hline & Moderate & $|6.55|$ & 8.676 & 98 \\
\hline & Flourishing & 6.444 & 5.642 & 216 \\
\hline & Total & 10.356 & 9.105 & 326 \\
\hline \multirow[t]{4}{*}{ Anxiety } & Languishing & 22.333 & 10.508 & 12 \\
\hline & Moderate & 14.612 & 9.019 & 98 \\
\hline & Flourishing & 8.231 & 6.901 & 216 \\
\hline & Total & 10.669 & 8.555 & 326 \\
\hline \multirow[t]{5}{*}{ Stress } & Languishing & 29.333 & 7.691 & 12 \\
\hline & Moderate & I7.286 & 8.908 & 98 \\
\hline & Flourishing & 8.315 & 6.937 & 216 \\
\hline & Total & 11.785 & 9.274 & 326 \\
\hline & Employment Status & $M$ & $S D$ & $N$ \\
\hline \multirow[t]{5}{*}{ Depression } & Full-time Contractual & 6 & 2.828 & 4 \\
\hline & Full-time Probationary & 13.575 & 10.497 & 127 \\
\hline & Full-time Regular & 8.286 & 7.462 & 189 \\
\hline & Part-time & 10.333 & 8.524 & 6 \\
\hline & Total & 10.356 & 9.104 & 326 \\
\hline \multirow[t]{5}{*}{ Anxiety } & Full-time Contractual & 8.5 & 5.744 & 4 \\
\hline & Full-time Probationary & 12.898 & 9.457 & 127 \\
\hline & Full-time Regular & 9.111 & 7.395 & 189 \\
\hline & Part-time & 14 & 13.856 & 6 \\
\hline & Total & 10.669 & 8.555 & 326 \\
\hline \multirow[t]{6}{*}{ Stress } & Full-time Contractual & II & 5.033 & 4 \\
\hline & Full-time Probationary & |4.583 & $10.21 \mid$ & 127 \\
\hline & Full-time Regular & 9.852 & 8.094 & 189 \\
\hline & Part-time & 14 & II.798 & 6 \\
\hline & Total & 11.785 & 9.275 & 326 \\
\hline & Position & $M$ & $S D$ & $N$ \\
\hline \multirow[t]{4}{*}{ Depression } & Admin & 9.7 & 8.951 & 20 \\
\hline & Faculty & II.474 & 9.851 & 228 \\
\hline & NTP & 7.256 & 5.518 & 78 \\
\hline & Total & 10.356 & 9.105 & 326 \\
\hline \multirow[t]{4}{*}{ Anxiety } & Admin & 11.0000 & 9.459 & 20 \\
\hline & Faculty & $|1.56| 4$ & 9.274 & 228 \\
\hline & NTP & 7.9744 & 4.851 & 78 \\
\hline & Total & 10.6687 & 8.555 & 326 \\
\hline \multirow[t]{3}{*}{ Stress } & Admin & 10.9 & 8.571 & 20 \\
\hline & Faculty & 12.947 & 10.108 & 228 \\
\hline & NTP & 8.615 & 5.416 & 78 \\
\hline
\end{tabular}




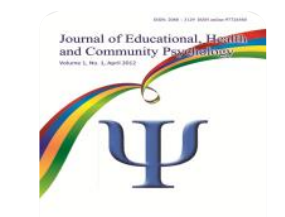

Journal of Educational, Health and Community

Psychology (Vol 11, No 1, 2022 E-ISSN 2460-8467)

\begin{tabular}{lllll} 
& Total & 11.785 & 9.274 & 326 \\
\hline Depression & Marital Status & $M$ & $S D$ & $N$ \\
& Common-Law & 4 & 3.266 & 4 \\
& Legally Separated & 2 & 2 & 3 \\
& Married & 7.876 & 7.32 & 161 \\
& Never Married & 13.507 & 10.191 & 146 \\
& Separated & 12.333 & 5.989 & 6 \\
& Widowed & 6.667 & 5.888 & 6 \\
& Total & 10.356 & 9.105 & 326 \\
\hline Anxiety & Common-Law & 7.5 & 4.123 & 4 \\
& Legally Separated & 10 & 8.718 & 3 \\
& Married & 9.217 & 7.537 & 161 \\
& Never Married & 12.26 & 9.498 & 146 \\
& Separated & 15.67 & 8.335 & 6 \\
& Widowed & 8.333 & 5.125 & 6 \\
& Total & & & 326 \\
& & 10.669 & 8.555 & \\
\hline Stress & Common-Law & 5.5 & 3.416 & 4 \\
& Legally Separated & 5.333 & 4.619 & 3 \\
& Married & 9.851 & 8.374 & 161 \\
& Never Married & 14.164 & 9.931 & 146 \\
& Separated & 16.333 & 6.86 & 6 \\
Widowed & 8.667 & 6.772 & 6 \\
& Total & 11.785 & 9.275 & 326 \\
\hline
\end{tabular}

In table 4, employees with languishing mental health obtained extremely severe levels of depression and anxiety; and severe levels of stress. Employees with Flourishing mental health reported normal levels of depression and stress; and mild anxiety. For the employment status, Full-time probationary employees reported a higher level albeit the normal levels of depression and anxiety; and mild stress with part-time, full-time, regular, and full-time contractual employees. Non-teaching personnel (NTP) obtained the lowest level with normal levels of depression and stress; mild anxiety. Faculty members reported the highest levels with a mild level of depression and stress; and moderate stress. For marital status, employees who were never married reported the highest level in their group with mild levels of depression. Meanwhile, employees who are separated from their spouses reported the highest levels of anxiety and stress with severe and mild levels, respectively. 


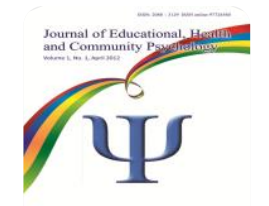

Journal of Educational, Health and Community

Psychology (Vol 11, No 1, 2022 E-ISSN 2460-8467)

Table 5

Multivariate Test of Common Mental Health Symptoms according to Mental Health, Employment Status, Employee Position, and Marital Status

\begin{tabular}{llllll}
\hline & Wilk's $\Lambda$ & $\mathrm{F}$ & $\mathrm{dfl}$ & $\mathrm{df2}$ & partial $\eta 2$ \\
\hline Mental Health & .552 & $37.053^{* * *}$ & 6 & 642. & .257 \\
Employment Status & .904 & $3.653^{* * *}$ & 9 & 778.947 & .033 \\
Position & .955 & $2.482^{*}$ & 6 & 642 & .023 \\
Marital Status & .864 & $3.179^{* * *}$ & 15 & 878.259 & .047 \\
\hline
\end{tabular}

Note: $\mathrm{p}<.05^{*}, \mathrm{p}<.01 * *, \mathrm{p}<.001 * * *$

Employee mental health status, employment status, and position have obtained significant differences in their depression, anxiety, and stress levels. While employee marital status only obtained significant differences in their depression and stress levels (table 6).

Table 6

Tests of Between-Subjects Effects of Common Mental Health Symptoms according to Employment Status, Employee Position, and Marital Status

\begin{tabular}{|c|c|c|c|c|c|c|c|}
\hline & $\begin{array}{l}\text { Dependent } \\
\text { Variable }\end{array}$ & SS & $\mathrm{df}$ & $\begin{array}{l}\text { Mean } \\
\text { Square }\end{array}$ & $\mathrm{F}$ & $\mathrm{P}$-value & $\begin{array}{l}\text { partial } \\
\text { n2 }\end{array}$ \\
\hline \multirow{3}{*}{$\begin{array}{l}\text { Mental Health } \\
\text { Continuum }\end{array}$} & Depression & 11775.479 & 2 & 5887.740 & 125.385 & .000 & .437 \\
\hline & Anxiety & 4439.863 & 2 & 2219.931 & 37.063 & .000 & .187 \\
\hline & Stress & 9261.710 & 2 & 4630.855 & 80.008 & .000 & .331 \\
\hline \multirow{3}{*}{$\begin{array}{l}\text { Employment } \\
\text { Status }\end{array}$} & Depression & 2201.780 & 3 & 733.927 & 9.552 & .000 & .082 \\
\hline & Anxiety & I I 74.885 & 3 & 391.628 & 5.577 & .001 & .049 \\
\hline & Stress & 1732.236 & 3 & 577.412 & 7.090 & .000 & .062 \\
\hline \multirow[t]{3}{*}{ Position } & Depression & 1042.810 & 2 & 521.405 & 6.502 & .002 & .039 \\
\hline & Anxiety & 750.132 & 2 & 375.066 & 5.259 & .006 & .032 \\
\hline & Stress & 1107.339 & 2 & 553.670 & 6.661 & .001 & .040 \\
\hline \multirow[t]{3}{*}{ Marital Status } & Depression & 2916.049 & 5 & 583.210 & 7.767 & .000 & .108 \\
\hline & Anxiety & 933.053 & 5 & $|86.61|$ & 2.613 & .025 & .039 \\
\hline & Stress & 1894.159 & 5 & 378.832 & 4.651 & .000 & .068 \\
\hline
\end{tabular}

Note: Bonferoni correction - significant at $p<.025$ 


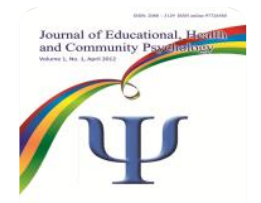

The Tukey posthoc analysis (table 7) revealed that mean scores for Depression, Anxiety and Stress obtained significant difference between all classifications of mental health, with Languishing being significantly different to Moderate and Flourishing Mental Health, and Moderate Mental Health to Flourishing according the level of depression, anxiety, and stress. Employees with Languishing mental health obtained highest levels of depression, anxiety, and stress from severe to extremely serve. While, flourishing with the lowest level with normal to mild level (see table 4 for split descriptive).

Table 7

Comparison Between Mean Differences of Common Mental Health Symptoms according to Mental Health Category

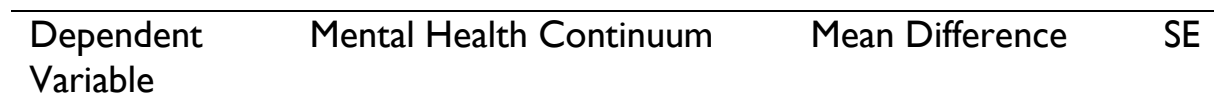

\begin{tabular}{lllll}
\hline Depression & Languishing & Moderate & $13.616^{* * *}$ & 2.095 \\
& & Flourishing & $23.722^{* * *}$ & 2.032 \\
\cline { 2 - 5 } & Moderate & Flourishing & $10.106^{* * *}$ & .835 \\
\hline Anxiety & Languishing & Moderate & $7.721^{* *}$ & 2.367 \\
& & Flourishing & $14.102^{* * *}$ & 2.295 \\
\cline { 2 - 5 } & Moderate & Flourishing & $6.381^{* * *}$ & .942 \\
\hline Stress & Languishing & Moderate & $12.048^{* * *}$ & 2.327 \\
& & Flourishing & $21.018^{* * *}$ & 2.256 \\
\cline { 2 - 5 } & Moderate & Flourishing & $8.971^{* * *}$ & .926 \\
\hline
\end{tabular}

Note: $\mathrm{p}<.05 *, \mathrm{p}<.01 * *, \mathrm{p}<.001 * *$

In table 8, depression, anxiety, and stress of Full-time Regular and Full-time Probationary employees are significantly different. Full-time Probationary employees expressed higher levels of common mental health symptoms, ranging from mild to moderate (see table 4 for split descriptive). 
Table 8

Comparison Between Mean Differences of Common Mental Health Symptoms according to Employment Status

\section{Dependent}

\begin{tabular}{|c|c|c|c|c|}
\hline \multirow{2}{*}{$\begin{array}{l}\text { Variable } \\
\text { Depression }\end{array}$} & \multicolumn{2}{|c|}{ Employment Status } & Mean Difference & SE \\
\hline & $\begin{array}{l}\text { Full-time } \\
\text { Contractual }\end{array}$ & $\begin{array}{l}\text { Full-time } \\
\text { Probationary }\end{array}$ & -7.575 & $4.45 I$ \\
\hline & & $\begin{array}{l}\text { Full-time } \\
\text { Regular }\end{array}$ & -2.286 & 4.429 \\
\hline & & Part-time & -4.333 & 5.658 \\
\hline & $\begin{array}{l}\text { Full-time } \\
\text { Probationary }\end{array}$ & $\begin{array}{l}\text { Full-time } \\
\text { Regular }\end{array}$ & $5.289 * * *$ & 1.006 \\
\hline & & Part-time & 3.241 & 3.662 \\
\hline & Full-time & Part-time & & 3.635 \\
\hline & Regular & & -2.048 & \\
\hline \multirow[t]{6}{*}{ Anxiety } & $\begin{array}{l}\text { Full-time } \\
\text { Contractual }\end{array}$ & $\begin{array}{l}\text { Full-time } \\
\text { Probationary }\end{array}$ & -4.398 & 4.255 \\
\hline & & $\begin{array}{l}\text { Full-time } \\
\text { Regular }\end{array}$ & -.611 & 4.234 \\
\hline & & Part-time & -5.5 & 5.409 \\
\hline & $\begin{array}{l}\text { Full-time } \\
\text { Probationary }\end{array}$ & $\begin{array}{l}\text { Full-time } \\
\text { Regular }\end{array}$ & $3.786^{* * *}$ & .961 \\
\hline & & Part-time & -1.102 & 3.501 \\
\hline & $\begin{array}{l}\text { Full-time } \\
\text { Regular }\end{array}$ & Part-time & -4.888 & 3.475 \\
\hline \multirow[t]{6}{*}{ Stress } & $\begin{array}{l}\text { Full-time } \\
\text { Contractual }\end{array}$ & $\begin{array}{l}\text { Full-time } \\
\text { Probationary }\end{array}$ & -3.583 & 4.582 \\
\hline & & $\begin{array}{l}\text { Full-time } \\
\text { Regular }\end{array}$ & 1.148 & 4.559 \\
\hline & & Part-time & -3 & 5.825 \\
\hline & $\begin{array}{l}\text { Full-time } \\
\text { Probationary }\end{array}$ & $\begin{array}{l}\text { Full-time } \\
\text { Regular }\end{array}$ & $4.73 I^{* * * *}$ & 1.035 \\
\hline & & Part-time & .583 & 3.77 \\
\hline & $\begin{array}{l}\text { Full-time } \\
\text { Regular }\end{array}$ & Part-time & $-4 .|48|$ & 3.742 \\
\hline
\end{tabular}

Note: $\mathrm{p}<.05^{*}, \mathrm{p}<.0 \mathrm{I}^{* *}, \mathrm{p}<.00 \mathrm{I}^{* * * *}$ 


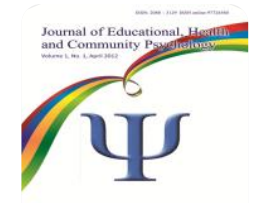

\section{Journal of Educational, Health and Community \\ PSychology (Vol 11, No 1, 2022 E-ISSN 2460-8467)}

Table 9

Comparison Between Mean Differences of Common Mental Health Symptoms according to Position

\begin{tabular}{lllll}
$\begin{array}{l}\text { Dependent } \\
\text { Variable }\end{array}$ & Position & & Mean Difference & SE \\
\hline Depression & Admin & Faculty & -1.774 & 2.0883 \\
& & NTP & 2.443 & 2.244 \\
\cline { 2 - 5 } & Faculty & NTP & $4.217^{* *}$ & 1.175 \\
\hline Anxiety & Admin & Faculty & -.561 & 1.969 \\
& & NTP & 3.026 & 2.1167 \\
\cline { 2 - 5 } & Faculty & NTP & $3.587^{*}$ & 1.108 \\
\hline Stress & Admin & Faculty & -2.047 & 2.126 \\
& & NTP & 2.285 & 2.285 \\
\hline & Faculty & NTP & $4.332^{* *}$ & 1.196
\end{tabular}

Note: $\mathrm{p}<.05^{*}, \mathrm{p}<.0 I^{* *}, \mathrm{p}<.00 I^{* * *}$

In terms of position (Table 9), significant differences between Faculty and Non-teaching personnel (NTP) have been observed with Faculty members experiencing higher levels of common mental health symptoms, ranging from mild to moderate (see table 4 for split desciptives).

Table 10

Comparison Between Mean Differences of Common Mental Health Symptoms according to Marital Status

\begin{tabular}{lllll}
$\begin{array}{llll}\text { Dependent } \\
\text { Variable }\end{array}$ & $\begin{array}{l}\text { Marital } \\
\text { Status }\end{array}$ & Marital Status & Mean Difference & SE \\
\hline Depression & Common- & Legally Separated & 2 & 6.618 \\
& Law & Married & -3.876 & 4.386 \\
& & Never Married & -9.507 & 4.391 \\
& & Separated & -8.333 & 5.59 \\
& & Widowed & -2.667 & 5.593 \\
\cline { 2 - 5 } & Legally & Married & -5.876 & 5.0492 \\
& Separated & & -11.507 & 5.053 \\
& & Never Married & -10.333 & 6.127 \\
& & Separated & -4.667 & .9 .127 \\
\cline { 2 - 5 } & Married & Never Married & $-5.631 I^{* * *}$ & 3.602
\end{tabular}



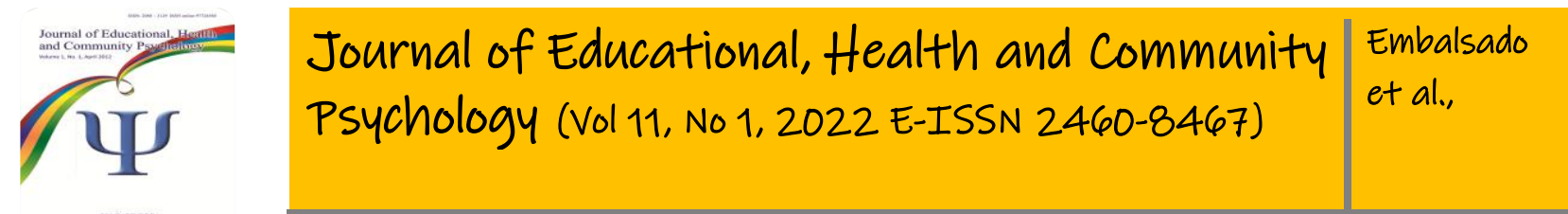

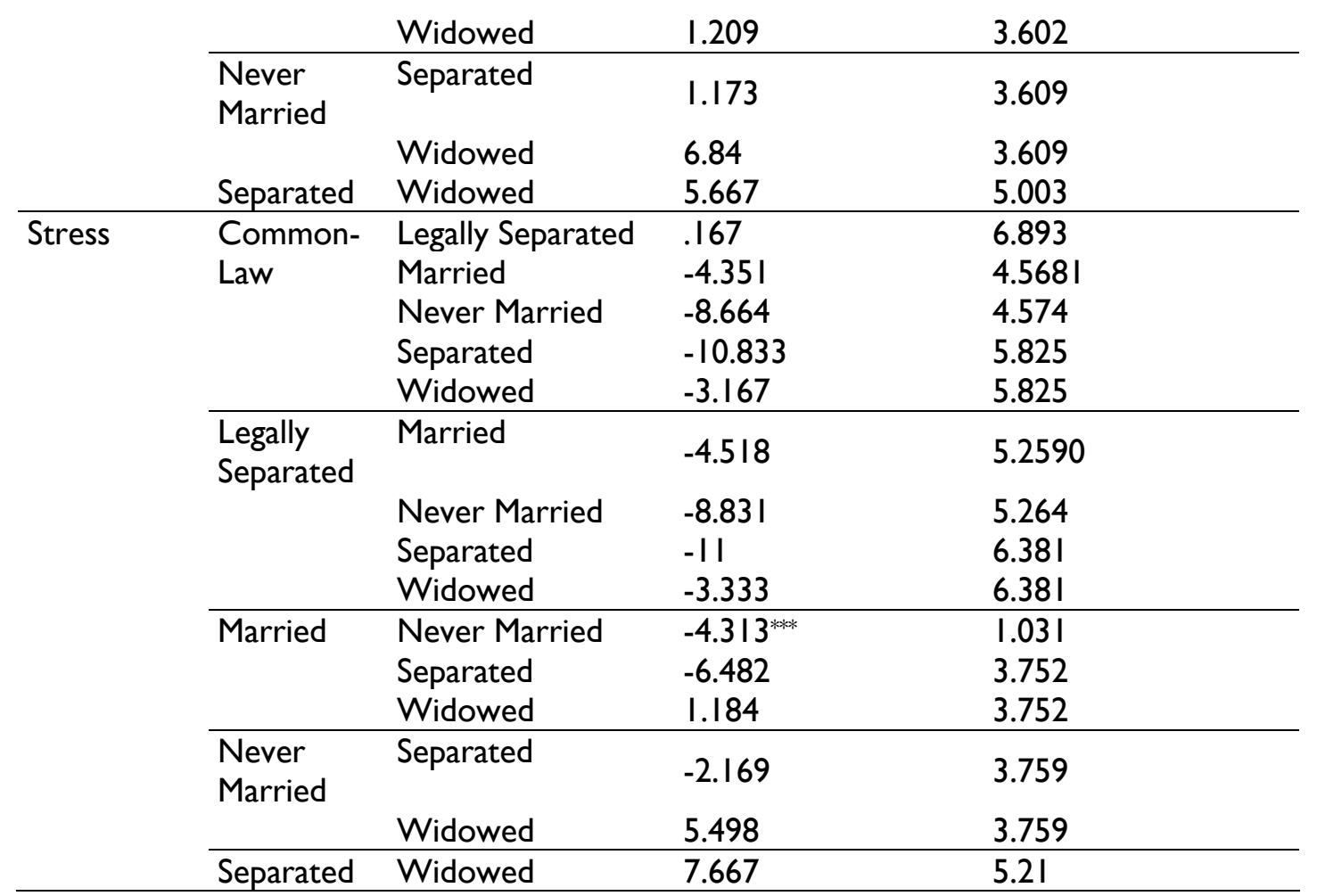

Note: $\mathrm{p}<.05^{*}, \mathrm{p}<.0 I^{* *}, \mathrm{p}<.00 I^{* * * *}$

Table 10 shows the significant difference between married and never married employees, never married employees reported higher levels of depression, and stress than married employees, they experienced moderate levels (see table 4 split descriptive statistics).

\section{Discussion}

The study intends to explore if the employee's well-being determines their depression, anxiety, and stress levels. The results show that the employee's psychological and emotional well-being inversely predicts depression, anxiety, and stress; an increase in their well-being leads to lower levels of depression, anxiety, and stress. A significant difference with the common mental health symptoms of employees according to mental health category, employment status and position, and marital status was also found. 


\section{Employee Well-being as Determinants of Common Mental Health Symptoms}

Psychological well-being inversely predicts common mental symptoms (depression, anxiety, and stress). Psychological well-being was also the best determinant of employee depression and stress. Results support existing studies with psychological well-being's inverse association with depression, anxiety, and stress (Daniels \& Guppy, 1994; Liu et al., 2009; Wood \& Joseph, 2010; Terry et al., 1993). The disruption caused by COVID-19 influenced the employees' psychological well-being as it affects their optimal functioning in fulfilling their duties (Cleland et al., 2020; Hindman \& Bustamante, 2019; Roberts et al., 2016). Universities face an unfamiliar work environment by shifting to full-online classes making educators and staff more vulnerable to common mental health symptoms (Cleland et al., 2020; Talidong \& Toquero, 2020). Even though universities provide training and support for educators and staff the abrupt transition hamper their effective adaptation in using educational resources, online teaching, use of technology, and communication (Atiles et al., 202I; Kosir et al., 2020). Hampering employee functioning affects psychological well-being making them unproductive and experience depression, anxiety, and stress symptom (Ceri \& Cicek, 202I; lasevoli et al., 2020; Rajkumar, 2020). Instability with the environment can harm the employee's environmental mastery, inhibiting them to manage their environment according to their needs since they have to manage between occupational and personal demands in the pandemic (Ceri \& Cicek, 202I; Knoesen, \& Naude, 2017; Ryff, 1989; Ryff \& Keyes, 1995). Not only with environmental mastery but all elements of psychological well-being have been affected during the pandemic (Ceri \& Cicek, 202I; lasevoli et al., 2020; Rajkumar, 2020).

Emotional well-being inversely predicts common mental symptoms (depression, anxiety, and stress). Emotional well-being predicted employees' anxiety the most. Health protocols that affect the personal sense of freedom influence emotional well-being, people tend to be less satisfied with life and experience negative effects when prohibited to go out of their homes (Pfefferbaum \& North, 2020). Not only health protocols but even occupational demands can affect an individual's personal sense of freedom. The shift from face-to-face classes to full-time online education disrupts the daily habits of educators because educators tend to overextend working on teaching preparations (Cleland et al., 2020). The decrease in emotional well-being caused by the pandemic heightens anxiety levels and other common mental health 
symptoms. The uncertain situation as seen by the shortage of testing and treatment, financial problems, inconsistent and vague information from authorities accounts for lower emotional well-being (Lades et al. 2020; Langeland, 2014; Pfefferbaum \& North, 2020; Restubog et al., 2020; Yang \& Ma, 2020).

Social well-being failed to predict common mental health symptoms. Results indicate that an individual's perception and experiences of different social events and responses to social challenges (Keyes, 1998) and even though support from peers and supervisors are important in maintaining positive mental health (Collie et al., 2017), social well-being failed to predict the depression, anxiety, and stress levels of university employees. The health protocols. Implementation of strict protocols decreased the time spent outdoors, and shifting to a work-from-home set-up contributes to mental health issues (Lades et al., 2020; Pfefferbaum \& North, 2020). This shift leads to social changes contributing to mental health problems (Saladin et al., 2020). However, even isolated from peers, people continuously interact virtually through social media and other platforms. Even physically isolated, online interactions can help foster the connection (Van Bavel et al, 202I). It is also important to consider demographic profiles, personal and family characteristics (Misheva, 2015) since the effects of social media and online interaction can vary among ages and usage (Bekalu et al., 2019).

\section{Differences in Common Mental Health Symptoms}

The significant difference in the levels of depression, anxiety, and stress according to the mental health category shows that individuals who are experiencing languishing mental health are more likely to experience severe levels of depression, anxiety, and stress. As expected those individuals experiencing moderate and flourishing mental health reported normal to moderate levels of stress. The findings are in line with existing studies on the influence of mental health and common mental health symptoms. A study conducted with postdoctoral fellows also found a significant difference in the depression, anxiety, and stress levels among those with flourishing and languishing mental health (Gloria \& Steinhardt, 2013). It seems that an increase in mental health leads to a decrease in common mental health symptoms. Flourishing individuals experience fewer common mental health symptoms while languishing individuals may develop more symptoms that affect then daily functioning (Keyes, \& Brim, 2002; Keyes, 1998). 
Full-time regular employees experienced significantly lower levels of depression, anxiety, and stress than full-time probationary employees. Findings are consistent with existing studies. Tenured employees experience job security and stability which result to lower mental health issues (Posel et al., 202I; Ruffolo et al, 202I). The security of being tenured helps employees face the instability of the pandemic compared to probationary employees wherein economic challenges have to arise (Goldfarb et al., 202I; Hamouche, 2020). Meanwhile, Faculty members reported significantly high levels of depression, anxiety, and stress compared to NTPs. Faculty members are expected to provide instruction and accommodate the wellbeing of their students while managing their stress (Cleland et al, 2020; Minkos \& Gelbar, 2020). Educators tend to over-extend their working hours to prepare for their classes making them feel exhausted (Cleland et al., 2020). The implementation of full-online classes required different educational needs, requiring educators to adapt and implement a relevant curriculum in a short period (Hindman \& Bustamante, 2019; Tan et al., 2020), such as using and adapting to new technology to cater to educational needs while preparing for their classes (Atiles et al., 202I; Kosir et al., 2020).

Married employees reported lower levels of depression, anxiety, and stress compared to an employee who never married. Findings are consistent with married individuals reporting lower levels of mental health issues (Brown et al., 2005; Pieh et al., 2020; Williams et al., 2017). According to Brown et al. (2005), non-married individuals tend to have fewer economic resources, less social support, and poor physical health resulting in poor mental health. And receiving social support serves as a social safety net that can help married individuals to experience less common mental health symptoms (Williams et al., $2017)$.

\section{Implications}

The study has practical and theoretical contributions. We have expanded the literature on employee mental health using psychological, emotional, and social well-being as determinants of depression, anxiety, and stress within the academe. We demonstrated the significant role of the mental health continuum to 

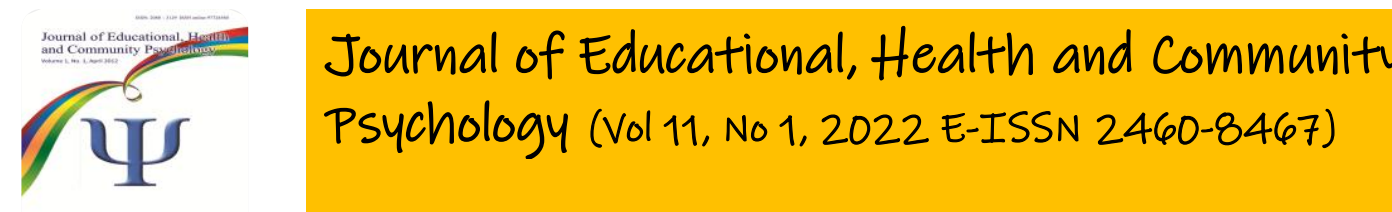

grasp a complete understanding of mental health and mental health issues. Scholars may explore possible mediating, or moderating variables to explain the mechanisms between mental health and mental health issues. They may conduct longitudinal studies to track the development of mental health during the pandemic. Furthermore, a qualitative study can be conducted to explore the experiences and insights of educators and staff in dealing with their mental health.

In practice, the study also has implications for educators and educational institutions. Educators may continuously reach out to there if they are facing difficulties in dealing with online education. They can also set specific logout times to avoid extended working hours. While, educational institutions may provide more flexible work hours, provide a manageable workload, and provide e-learning training to maximize the productivity of employees. Institutions can also provide educators basic training on how to deal with the personal stress and mental health issues of students.

\section{Conclusion}

In conclusion, employee's well-being significantly determines their depression, anxiety, and stress levels. Psychological well-being having an inverse relationship with all common mental health symptoms. Psychological well-being was also the best determinant of depression and stress. This shows that optimal functioning and learning how to manage the environment according to one's needs affects the development of mental health symptoms. Emotional well-being has an inverse relationship with all common mental health symptoms and also be the best determinant of stress. The pandemic leads to uncertainty affecting life satisfaction and increasing negative affect, leading to the development of mental health issues. The results with social well-being failed to support the hypothesis. Even isolated, people maintain social connections using social media and other platforms which opens the discussion for control variables that can explain their association. The significant difference with the levels of mental health according to the mental health category reflects show an increase in mental health would lead to a decrease in common mental symptoms. Tenured employees tend to experience lower levels of common health symptoms, while faculty members are more likely to experience high levels than NTPs. 
Married employees expressed lower levels of common mental health symptoms than employees who have not married.

\section{References}

Arslan, G., Yıldırım, M., Karataș, Z., Kabasakal, Z., \& Kılınç, M. (2020). Meaningful Living to Promote Complete Mental Health Among University Students in the Context of the COVID-19 Pandemic. International Journal of Mental Health and Addiction, I-I3. https://doi.org/I0.1007/s I |469-02000416-8

Atiles, J. T., Almodóvar, M., Chavarría Vargas, A., Dias, M. J. A., \& Zúñiga León, I. M. (202I). International responses to COVID-19: challenges faced by early childhood professionals. European Early Childhood Education Research Journal. https://doi.org/10.1080/1350293X.2021.1872674

Banna, M. H. Al, Sayeed, A., Kundu, S., Christopher, E., Hasan, M. T., Begum, M. R., Kormoker, T., Dola, S. T. I., Hassan, M. M., Chowdhury, S., \& Khan, M. S. I. (2020). The impact of the COVID-19 pandemic on the mental health of the adult population in Bangladesh: a nationwide cross-sectional study. International Journal of Environmental Health Research, 00(00), I-I2. https://doi.org// 0.1080/09603 I23.2020.1802409

Bekalu, M. A., McCloud, R. F., \& Viswanath, K. (2019). Association of Social Media Use With Social WellBeing, Positive Mental Health, and Self-Rated Health: Disentangling Routine Use From Emotional Connection to Use. Health Education \& Behavior, 46(2_suppl), 69S-80S. https://doi.org/I0.1 I77/1090198119863768

Berndt, E. R., Bailit, H. L., Keller, M. B., Verner, J. C., \& Finkelstein, S. N. (2000). Health care use and atwork productivity among employees with mental disorders. Health Affairs, 19(4), 244-256. https://doi.org//0.1377/hlthaff.19.4.244

Bolo, C., Sareen, J., Patten, S., Schmitz, N., Currie, S., \& Wang, J. (20I3). Receiving workplace mental health accommodations and the outcome of mental disorders in employees with a depressive and/or anxiety disorder. Journal of Occupational and Environmental Medicine, 55(II), I293-1299. https://doi.org/ I0.1097/JOM.0b0I3e31829fd065

Boreham, P., Povey, J., \& Tomaszewski, W. (2015). Work and social well-being: the impact of employment conditions on quality of life. The International Journal of Human Resource Management, 27(6), 593-6 II. http://dx.doi.org/l0.1080/09585192.2015.1027250

Brown, S. L., Bulanda, J. R., \& Lee, G. R. (2005). The significance of nonmarital cohabitation: Marital status and mental health benefits among middle-aged and older adults. Journals of Gerontology - Series B Psychological Sciences and Social Sciences, 60(I), 2 I-29. https://doi.org/I0.1093/geronb/60.I.S2I

Ceri, V., \& Cicek, I. (202I). Psychological Well-Being, Depression, and Stress During COVID-19 Pandemic in Turkey: A Comparative Study of Healthcare Professionals and Non-Healthcare 
Professionals. Psychology, Health \& Medicine, 26(I), 85-97. https://doi.org/10.1080/I3548506.2020.1859566

Cicognani, E. (2014). Social Well-Being. Encyclopedia of Quality of Life and Well-Being Research, 6193-6197. https://doi.org// 0.1007/978-94-007-0753-5_2797

Cleland, J., McKimm, J., Fuller, R., Taylor, D., Janczukowicz, J., \& Gibbs, T. (2020). Adapting to the impact of COVID-19: Sharing stories, sharing practice. Medical Teacher, 42(7), 772-775. https://doi.org//0.1080/0142159X.2020.1757635.

Collie, R. J., Martin, A. J., Bottrell, D., Armstrong, D., Ungar, M., \& Liebenberg, L. (2017). Social support, academic adversity and academic buoyancy: A person-centred analysis and implications for academic outcomes. Educational Psychology, 37, 550-564.

Daniels, K., \& Guppy, A. (1994). Occupational Stress, Social Support, Job Control, and Psychological Well-Being. Human Relations, 47(I2), I523-1544. https://doi.org/I0.1 I77/00I87267940470I 205.

Ferguson, K., Mang, C., \& Frost, L. (2012). Teacher Stress and Social Support Usage. Brock Education Journal, 26(2), 62-86.

Gloria, C.T., \& Steinhardt, M.A. (2013). Flourishing, languishing, and depressed postdoctoral fellows:

Differences in stress, anxiety, and depressive symptoms. Journal of Postdoctoral Affairs, 3(I), I-8.

Goldfarb, Y., Gal, E., \& Golan, O. (202I). Implications of Employment Changes Caused by COVID-19 on Mental Health and Work-Related Psychological Need Satisfaction of Autistic Employees: A MixedMethods Longitudinal Study. Journal of Autism and Developmental Disorders, 0123456789. https://doi.org/I 0. 1007/s 10803-02I-04902-3

Hamouche, S. (2020). COVID-19 and employees' mental health: stressors, moderators and agenda for $\begin{array}{lllll}\text { organizational } \quad \text { actions. Emerald } & \end{array}$ https://doi.org/I0.3524I/emeraldopenres. I3550.I

Hindman, A. H., \& Bustamante, A. S. (2019). Teacher depression as a dynamic variable: Exploring the nature and predictors of change over the head start year. Journal of Applied Developmental Psychology, $6 /$ (March 2019), 43-55. https://doi.org//0.1016/j.appdev.2018.09.004

lasevoli, F., Fornaro, M., D'Urso, G., Galletta, D., Casella, C., Paternoster, M., Buccelli, C., \& De Bartolomeis, A. (2020). Psychological distress in serious mental illness patients during the COVID19 outbreak and one-month mass quarantine in Italy. In Psychological Medicine (pp. I-3). Cambridge University Press. https://doi.org/I0.1017/S003329/72000I84I

Karaar, B., \& Canli, D. (2020). Psychological resilience and depression during the COVID-19 pandemic in Turkey. Psychiatria Danubina, 32(2), 273-279. https://doi.org/I0.24869/PSYD.2020.273

Keyes, C. L. M. (1998). Social Well-Being. Social Psychology Quarterly, 6I(2),I2I. https://doi.org// 0.2307/2787065.

Keyes, C. L. M., \& Brim, G. (2002). Keyes and human development program through mem-and lopez. Journal of Health and Social Research, 43(2), 207-222. 
Keyes, C. (2009). Overview of The Mental Health Continuum Short Form (MHC-SF). doi:I0.I3 I40/RG.2.2.24204.62088.

Knoesen, R., \& Naudé, L. (2018). Experiences of flourishing and languishing during the first year at university. Journal of Mental Health, 269-278. https://doi.org//0.1080/09638237.2017.1370635,

Košir, K., Dugonik, S., Huskić, A., Gračner, J., Kokol, Z., \& Krajnc, Z. (2020) Predictors of perceived teachers' and school counsellors' work stress in the transition period of online education in schools during the COVID-19 pandemic, Educational Studies, DOI: 10.1080/03055698.2020.1833840.

Lades, L., Laffan, K., Daly, M., \& Delaney, L. (2020). Daily emotional well-being during the COVID-I9 pandemic. British Journal Of Health Psychology, 25(4), 902-9 I I. https://doi.org/ I 0. I I I/bjhp. 12450

Langeland, E. (20I4). Emotional Well-Being. Encyclopedia Of Quality Of Life And Well-Being Research, I8741876. https://doi.org/10.1007/978-94-007-0753-5_859

Larson, J. S. (1996). The World Health Organization's definition of health: Social versus spiritual health. Social Indicators Research, 38(2), I8I-192. https://doi.org/10.1007/BF00300458.

Liu, Q., Shono, M., \& Kitamura, T. (2009). Psychological well-being, depression, and anxiety in Japanese university students. Depression and Anxiety, 26(8), E99-El05. https://doi.org// 0.1002/da.20455

Lovibond, S.H. \& Lovibond, P.F. (1995). Manual for the Depression Anxiety \& Stress Scales. (2nd Ed.)Sydney: Psychology Foundation.

Majumdar, P., Biswas, A., \& Sahu, S. (2020). COVID- 19 pandemic and lockdown: cause of sleep disruption, depression, somatic pain, and increased screen exposure of office workers and students of India. Chronobiology International, 00(00), I-I0. https://doi.org/I0.1080/07420528.2020.1786I07

Minkos, M. L., \& Gelbar, N. W. (202I). Considerations for educators in supporting student learning in the midst of COVID- 19. Psychology in the Schools, 58(2), 4l6-426. https://doi.org/10.1002/pits.22454

Misheva, V. (20I5). What Determines Emotional Well-Being? The Role of Adverse Experiences: Evidence Using Twin Data. Journal Of Happiness Studies, 17(5), 1921-1937. https://doi.org/10.1007/s109020I5-9678-9

Paulino, M., Dumas-Diniz, R., Brissos, S., Brites, R., Alho, L., Simões, M. R., \& Silva, C. F. (202I). COVID19 in Portugal: exploring the immediate psychological impact on the general population. Psychology, Health and Medicine, 26(I), 44-55. https://doi.org// 0.1080/I3548506.2020.1808236

Pfefferbaum, B., \& North, C. (2020). Mental Health and the Covid-19 Pandemic. New England Journal Of Medicine, 383(6), 510-5 I2. https://doi.org/10.1056/nejmp20080I7

Pieh, C., O'Rourke, T., Budimir, S., \& Probst, T. (2020). Relationship quality and mental health during COVID-1 9 lockdown. PLoS ONE, I-10. https://doi.org/I0.137//journal.pone.0238906

Plaisier, I., de Bruijn, J. G. M., de Graaf, R., Have, M. ten, Beekman, A. T. F., \& Penninx, B. W. J. H. (2007). The contribution of working conditions and social support to the onset of depressive and anxiety 
disorders among male and female employees. Social Science and Medicine, 64(2), 40I-4I0. https://doi.org/10.1016/j.socscimed.2006.09.008

Posel, D., Oyenubi, A., \& Kollamparambil, U. (202I). Job loss and mental health during the COVID- 19 lockdown: Evidence from South Africa. PLoS ONE, 16(3 March), I-I5. https://doi.org/I0.137I/journal.pone.0249352

Preece, M., Cayley, P. M., Scheuchl, U., \& Lam, R. W. (2006). The relevance of an employee assistance program to the treatment of workplace depression. Journal of Workplace Behavioral Health, $2 I(\mathrm{I})$, 67-77. https://doi.org//0.1300/J490v2In0I_06

Rajkumar, R. P. (2020). COVID- 19 and mental health: A review of the existing literature. Asian Journal of Psychiatry, 52(March), 102066. https://doi.org/I0.1016/j.ajp.2020.102066

Restubog, S., Ocampo, A., \& Wang, L. (2020). Taking control amidst the chaos: Emotion regulation during the COVID-19 pandemic. Journal of Vocational Behavior, I19, 103440. https://doi.org/10.1016/j.jvb.2020.103440

Roberts, A., LoCasale-Crouch, J., Hamre, B., \& DeCoster, J. (2016). Exploring Teachers' Depressive Symptoms, Interaction Quality, and Children's Social-Emotional Development in Head Start. Early Education and Development, 27(5), 642-654. https://doi.org/ I 0. I 080/10409289.20 I6. I I 27088

Roy, D., Tripathy, S., Kumar, S., Sharma, N., Verma, S. K., \& Kaushal, V. (2020). Study of knowledge, attitude, anxiety \& perceived mental healthcare need in Indian population during COVID-I9 pandemic. Asian Journal of Psychiatry, 5 I (I), 2020.

Ruffolo, M., Price, D., Schoultz, M., Leung, J., Bonsaksen, T., Thygesen, H., \& Geirdal, A. Ø. (202I). Employment Uncertainty and Mental Health During the COVID-19 Pandemic Initial Social Distancing Implementation: a Cross-national Study. Global Social Welfare, 8(2), I4I-I50. https://doi.org/10.1007/s40609-020-00201-4

Ryff, C. D. (1989). Happiness Is Everything, or Is It? Explorations on the Meaning of Psychological WellBeing. Journal of Personality and Social Psychology, 57(6), 1069-108I.

Ryff, C. D., \& Keyes, C. L. M. (1995). The Structure of Psychological Well-Being Revisited. 69(4), 719727.

Saladino, V., Algeri, D., \& Auriemma, V. (2020). The Psychological and Social Impact of Covid-19: New Perspectives of Well-Being. Frontiers in Psychology, II. https://doi.org/l0.3389/fpsyg.2020.577684.

Talidong, K. J. B., \& Toquero, C. M. D. (2020). Philippine Teachers' Practices to Deal with Anxiety amid COVID-19. Journal of Loss and Trauma, 25(6-7), 573-579. https://doi.org/10.1080/I5325024.2020.1759225

Tang, F., Liang, J., Zhang, H., Kelifa, M. M., He, Q., \& Wang, P. (202I). COVID-I 9 related depression and anxiety among quarantined respondents. Psychology and Health, 36(2), I64-I78. https://doi.org/10.1080/08870446.2020.1782410 
Terry, D. J., Nielsen, M., \& Perchard, L. (1993). Effects of work stress on psychological well-being and job satisfaction: The stress-buffering role of social support. Australian Journal of Psychology, 45(3), I68-175. https://doi.org/I0.1080/00049539308259/35

Thisted, C. N., Labriola, M., Vinther Nielsen, C., Kristiansen, S. T., Strøm, J., \& Bjerrum, M. B. (2020). Managing employees' depression from the employees', co-workers' and employers' perspectives. An integrative review. Disability and Rehabilitation, 42(4), 445-459. https://doi.org//0.1080/09638288.2018.1499823

Thorsteinsson, E. , Brown, R. \& Richards, C. (2014). The Relationship between Work-Stress, Psychological Stress and Staff Health and Work Outcomes in Office Workers. Psychology, 5, I30 I I3 I I. doi: 10.4236/psych.2014.5 I0141.

Van Bavel, J. J., Baicker, K., Boggio, P. S., Capraro, V., Cichocka, A., Cikara, M., Crockett, M. J., Crum, A. J., Douglas, K. M., Druckman, J. N. Drury, J., Dube, O., Ellemers, N., Finkel, E. J., Fowler, J. H., Gelfand, M., Han, S., Haslam, S. A., Jetten, J., Kitayama, S., Mobbs, D., Napper, L. E., Packer, D. J., Pennycook, G., Peters, E., Petty, R. E., Rand, D. G., Reicher, S. D., Schnall, S., Shariff, A., Skitka, L. J., Smith, S. S., Sunstein, C. R., Tabri, N., Tucker, J. A., van der Linden, S., Van Lange, P. A. M., Weeden, K. A., Wohl, M. J. A., Zaki, J., Zion, S. \& Willer, R. (202I).Using social and behavioural science to support COVID-19 pandemic response. Nature Human Behavior, 4, 460-47I. doi: 10.3 I 234/osf.io/y38m

Van, D., McLaws, M. L., Crimmins, J., Maclntyre, C. R., \& Seale, H. (2010). University life and pandemic influenza: Attitudes and intended behaviour of staff and students towards pandemic (HINI) 2009. BMC Public Health, I0. https://doi.org/I0.I I86/I47I-2458-10-I30

Vinkers, C. H., van Amelsvoort, T., Bisson, J. I., Branchi, I., Cryan, J. F., Domschke, K., Howes, O. D., Manchia, M., Pinto, L., de Quervain, D., Schmidt, M. V., \& van der Wee, N. (2020). Stress resilience during the coronavirus pandemic. European neuropsychopharmacology: the journal of the European College of Neuropsychopharmacology, 35, 12-16. https://doi.org/10.10 I6/j.euroneuro.2020.05.003

Westerhof, G. J., \& Keyes, C. L. M. (2010). Mental illness and mental health: The two continua model across the lifespan. Journal of Adult Development, I7(2), I I0-1 I9. https://doi.org/I0.1007/s I0804009-9082-y

Williams, L., Zhang, R., \& Packard, K. C. (2017). Factors affecting the physical and mental health of older adults in China: The importance of marital status, child proximity, and gender. SSM - Population Health, 20-36. https://doi.org/10.1016/j.ssmph.2016.11.005

Wood, A. M., \& Joseph, S. (2010). The absence of positive psychological (eudemonic) well-being as a risk factor for depression: A ten year cohort study. Journal of Affective Disorders, 122(3), 213-217. https://doi.org/10.1016/j.jad.2009.06.032 
Xu, X., Xue, K., Wang, L., Gursoy, D., \& Song, Z. (202I) Effects of customer-to-customer social interactions in virtual travel communities on brand attachment: The mediating role of social wellbeing. Tourism Management Perspectives, 38, 22 I I-9736. https://doi.org/ I0.1016/j.tmp.2021.100790

Yamaguchi, K., Takebayashi, Y., Miyamae, M., Komazawa, A., Yokoyama, C., \& Ito, M. (2020). Role of Focusing on the Positive Side During COVID-19 Outbreak: Mental Health Perspective From Positive Psychology. Psychological Trauma: Theory, Research, Practice, and Policy. https://doi.org//0.1037/tra0000807

Yang, H., \& Ma, J. (2020). How an Epidemic Outbreak Impacts Happiness: Factors that worsen (vs. protect) emotional well-being during the coronavirus pandemic. Psychiatry Research, 289, II 3045. https://doi.org//0.1016/j.psychres.2020.113045

Yenan Wang, Yu Di, Junjie Ye \& Wenbin Wei (2020): Study on the public psychological states and its related factors during the outbreak of coronavirus disease 2019 (COVID-19) in some regions of China, Psychology, Health \& Medicine, DOI: 10.1080/I3548506.2020.17468I7 